




2017-08-01

\section{Vibration diagnosis of a gearbox by wavelet bicoherence technology}

Gelman, Leonid

British Institute of Non-destructive Testing

Gelman L, Solinski K, Shaw B, Vaidhianathasamy M, Vibration diagnosis of a gearbox by wavelet bicoherence technology, Insight: Non-Destructive Testing \& Condition Monitoring, Vol. 59, Issue 8, August 2017, pp. 440-444

http://dx.doi.org/10.1784/insi.2017.59.8.440

Downloaded from Cranfield Library Services E-Repository 